\title{
Tailoring of Cortical Excisions for Frontal Lobe Epilepsy
}

\author{
Theodore Rasmussen
}

\begin{abstract}
Follow-up data of 2 to 49 years' duration are presented on 257 patients with cortical excisions for non-tumoral frontal lobe epilepsy. Twenty-six percent have become and remained seizure free. Another $30 \%$ have had a marked reduction of seizure tendency. Thus a total of $56 \%$ have had a long term complete or marked reduction of seizure tendency after the cortical resection. The cortical resections were classified into 5 regional categories. Forty-seven percent of patients with anterior frontal resections were in the seizure free category, as were $35 \%$ of patients with excisions limited to the convexity cortex and $32 \%$ of patients with maximal or near maximal frontal lobectomy. Only $18 \%$ of patients with frontal parasaggital excisions were in the seizure free category, and $10 \%$ of the patients who had excision of frontal cortex plus limited excision of adjacent central and/or temporal cortex.
\end{abstract}

RÉSUMÉ: Détermination de l'extension de la résection corticale dans l'épilepsie frontale. Deux cent cinquante sept patients ayant subi une excision corticale pour une épilepsie frontale d'origine autre que tumorale ont été suivis sur une période de 2 à 49 ans. Chez $26 \%$ des patients, les crises ont cessé, sans récidive. Chez 30\% des patients une diminution marquée des crises est survenue. Donc, au total, $56 \%$ des patients ont été guéris ou se sont améliorés de façon importante après la résection corticale. Les résections corticales frontales sont divisées en 5 catégories, selon la région frontale opérée. Quarante-sept pour cent des patients opérés dans la région frontale antérieure ont cessé d'avoir des crises. la proportion passant à $35 \%$ pour les chirurgies d'exérèse limitée à la convexité frontale et à $32 \%$ pour les patients soumis à une lobectomie frontale maximale ou quasi-maximale. Seulement $18 \%$ des patients avec excision frontale parasagittale se retrouvèrent dans la catégorie des patients guéris, le chiffre correspondant étant de $10 \%$ seulement dans les cas d'excision corticale frontale avec excision limitée du cortex central et/ou temporal avoisinant.

Can. J. Neurol. Sci. 1991; 18:606-610

Operations for frontal lobe epilepsy have constituted an important segment of the Montreal Neurological Institute (MNI) surgical seizure series from its beginnings with Dr. Wilder Penfield's first seizure operation in Montreal in the fall of 1928. Various aspects of the frontal lobe portion of the series have been reported periodically since $1930 .{ }^{1-28}$ This report will update some aspects of these reports and will analyze the effectiveness of various types of frontal excisions, carried out up to the end of 1980 , in reducing the patient's seizure tendencies.

It should be mentioned at the outset that in this series, the posterior border of the frontal lobe is considered to be the precentral sulcus, not the Rolandic fissure as is the classical neuroanatomical definition. The pre- and postcentral gyri are considered to be a separate functional unit because the results of both electrical stimulation and of discrete cortical excisions of these two gyri are so different from the adjacent frontal and parietal cortex.

By the end of 1980, 402 cases of frontal lobe epilepsy had accumulated in the series. These represent $18 \%$ of the total MNI surgical seizure series, 2177 patients at that time (Figure 1). These 402 patients include 119 patients with tumoral epilepto- genic lesions of the frontal lobe, predominantly indolent, slowly growing gliomas. These patients who masqueraded as ordinary seizure problems, and were operated upon as such, constitute $29 \%$ of the total frontal lobe series. They will not be considered further in this report, which will be limited to the remaining 283 patients with stable, non-tumoral epileptogenic lesions of the frontal lobe.

Post-natal trauma was the most common presumed etiological factor and involved 113 patients, $40 \%$ of the 283 patients with non-tumoral frontal epileptogenic lesions. Post-inflammatory brain scarring was the presumed cause in 39 patients, $14 \%$. Birth trauma was involved in 33 patients, $12 \%$. An interesting group of miscellaneous lesions, congenital and acquired, were present in 41 patients, $14 \%$. No etiological factors could be determined in 49 patients, $19 \%$, and multiple potential etiological factors were present in 8 patients, $3 \%$.

There is great variability in the location and size of the cortical excisions that have been carried out in these patients with frontal lobe epilepsy. Patient H.Pe, a 14-year-old girl, is representative of those with relatively small cortical excisions (Figure 2 ). She had somatosensori-motor seizures involving her left 


\begin{tabular}{|lr|}
\hline \multicolumn{2}{|c|}{$\begin{array}{c}\text { Montreal Neurological Institute Surgical Seizure Series } \\
\text { Anatomical Classification - patients operated upon } 1929 \text { through } 1980\end{array}$} \\
\hline Temporal lobe & $1210 \mathrm{pts}(56 \%)$ \\
Frontal lobe & $402 \mathrm{pts}(18 \%)$ \\
Central (sensorimotor) region & $151 \mathrm{pts}(7 \%)$ \\
Parietal lobe & $141 \mathrm{pts}(6 \%)$ \\
Occipital lobe & $30 \mathrm{pts}(1 \%)$ \\
Large multilobe lesions & $243 \mathrm{pts}(11 \%)$ \\
\hline & Total \\
\hline
\end{tabular}

Figure l. arm, increasing to an average of 5 per month at the time of her first operation.

Dr. Penfield operated upon her in 1929, well before the EEG era, and found a medium sized porencephalic cyst in the right frontal lobe. Electrical stimulation of the anterior margin of the cyst reproduced her habitual attack, so the anterior margin of the cyst was removed (stippled area). Her attacks recurred after 6 months, however, and persisted. Dr. Penfield reoperated in 1930, 18 months after the first operation and carried out a larger removal of the cortex around the cyst (blackened area). The second cortical excision resulted in a satisfactory reduction of her seizure tendency and she has been seizure free following the second operation to her last report in 1968,35 years postoperative, when she became lost to follow-up.

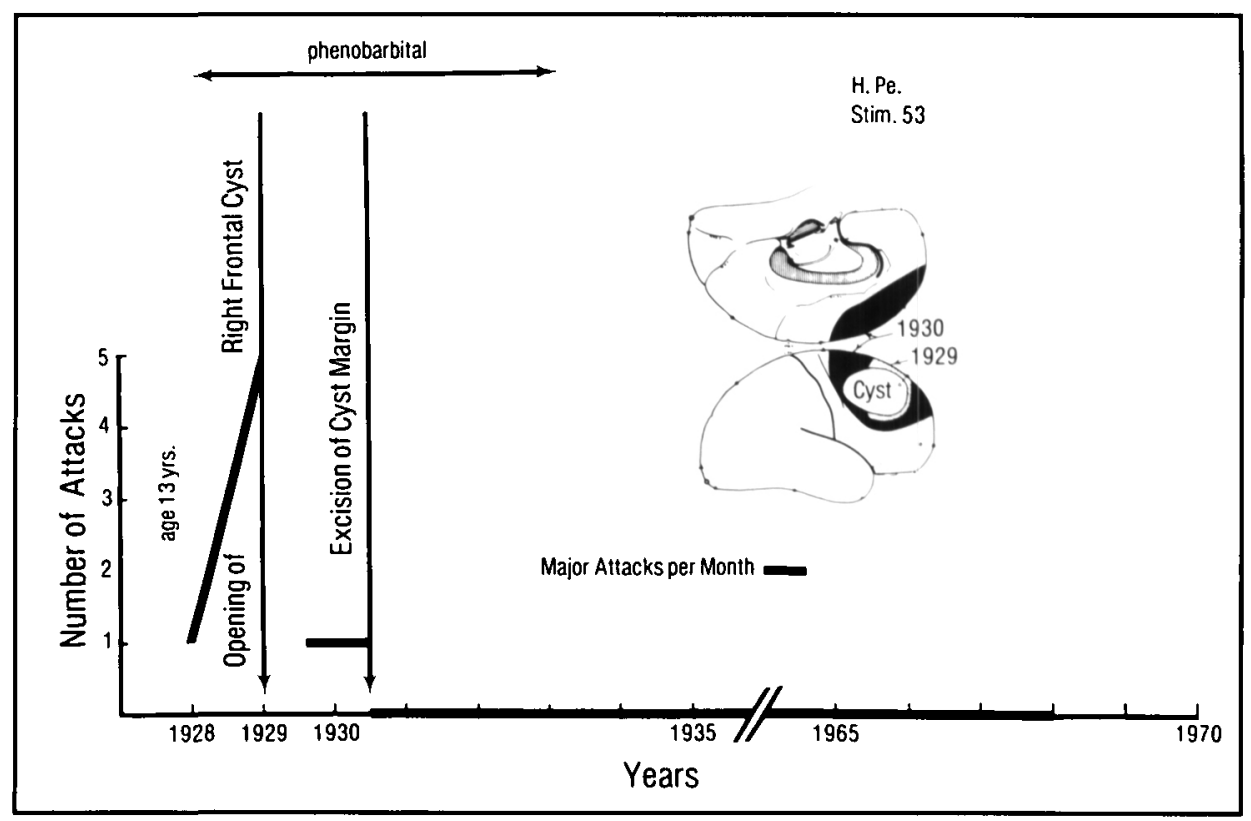

Figure 2 - Seizure frequency analysis, patient H.Pe. (from Rasmussen, reference 27)

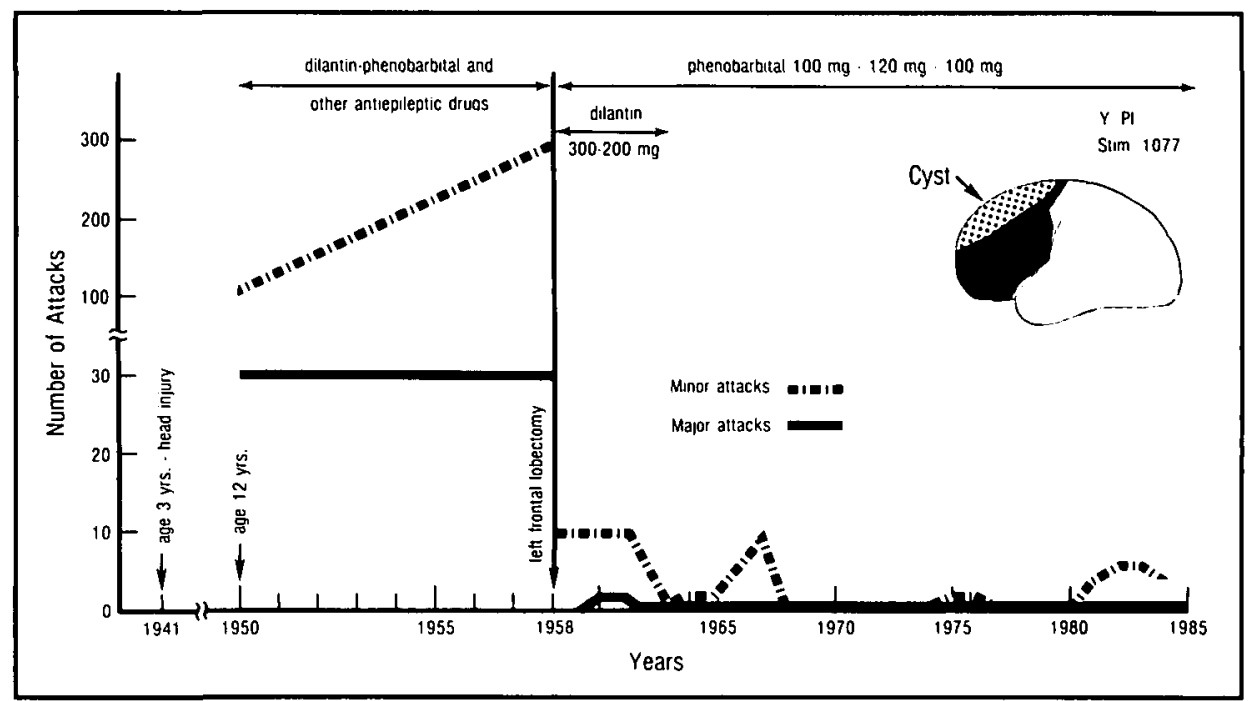

Figure 3-Seizure frequency analysis, patient Y.PI. 
A second patient, Y.Pl., provides an example of a large, maximal frontal excision (Figure 3). He is reported to have had a normal birth, but sustained a left sided head injury of undetermined severity at 3 years of age. He began to have both major and more frequent minor attacks at 13 years of age. When he was investigated at 20 years of age he was found to be moderately retarded with a full scale IQ of 69 , but the neurological examination was otherwise normal. X-ray studies were ambiguous but the EEG showed epileptiform spikes and spike-wave complexes over the entire left frontal lobe with some spread of the discharges to the right frontal lobe. Some independent spiking was also recorded over the left temporal region. A carotid amytal speech test proved that his speech functions were on the right, despite the fact that he was right handed.

A left frontal craniotomy in 1958 disclosed a large porencephalic cyst which had not been visualized in his preoperative pneumo-encephalogram. The cortical EEG showed spikes and spike-wave complexes over the frontal lobe, over the precentral gyrus and to a lesser degree below the fissure of Sylvius on the temporal lobe. The cortical excision ultimately included most of the face area of the precentral gyrus in addition to the entire frontal lobe.

He had a considerable right hemiparesis the next day but 2 weeks later his sensorimotor status had returned to his preoperative state except for a moderate droop and underaction of the right side of the mouth. His full scale IQ had risen from 69 preoperative to 79 at time of discharge and to 89 on a check-up 4 years postoperative.

After 2 major and somewhat more frequent brief minor motor attacks in his first 8 postoperative years, he had a long seizure free interval until 1980, 22 years postoperative. The brief minor attacks then recurred and have persisted at 1 to 3 month intervals to his last report in 1984, 26 years postoperative.

The overall results of these frontal cortical excisions on the seizure tendency can be evaluated in 257 of the total 283 patients (Figure 4). Sixty-eight patients (26\%) have become and remained seizure free (follow-up categories 0 and 1). Another 77 patients $(30 \%)$ have had a marked reduction of the seizure tendency (follow-up categories 2 and 3). Thus 56\% have had a complete or marked reduction of the seizure tendency following various types of frontal cortical excisions.

There were 112 patients in the less successful group, followup category 4 . As in the case of the temporal lobe series presented earlier at this session, some of these patients have had considerable benefit even though the operation failed to achieve the goal of reducing the patient's seizure tendency to a normal or near normal level. In nearly a third of these follow-up category 4 patients' the postoperative seizure rate has been about 1 to $10 \%$ of the preoperative rate. In another $1 / 3$ the rate has ranged between 10 and $50 \%$ of the preoperative rate. The remaining $1 / 3$ have had a lesser to no reduction.

Forty of the patients in the seizure free group (follow-up categories 0 and 1) who have been seizure free for 5 to 40 years (median 14 years) have been analyzed and reported recently as a "pure culture" of frontal lobe epilepsy. ${ }^{22}$ In 18 patients the anterior third half of the frontal lobe had been resected (Figure 5). Most of the frontal lobe was removed in 9 patients $(22.5 \%)$. Parasaggital excisions of various extent were carried out in 8 patients (20\%). Smaller cortical excisions limited to the convexity of the frontal lobe were carried out in the patients (12.5\%).

Typical examples of these types of cortical excisions, taken from operative photos and operative drainings, are shown in Figure 6. A fifth type of cortical excision, frontal plus, has been added for this analysis to include those patients whose cortical excisions, in addition to removal of frontal cortex, included limited excisions of adjacent central and/or temporal regions. Patient Y.PI., the second case presented, is a typical representation of this type of resection.

The results on the seizure tendency of these 5 types of cortical excisions are tabulated in Figure 7. Follow-up groups 0 and

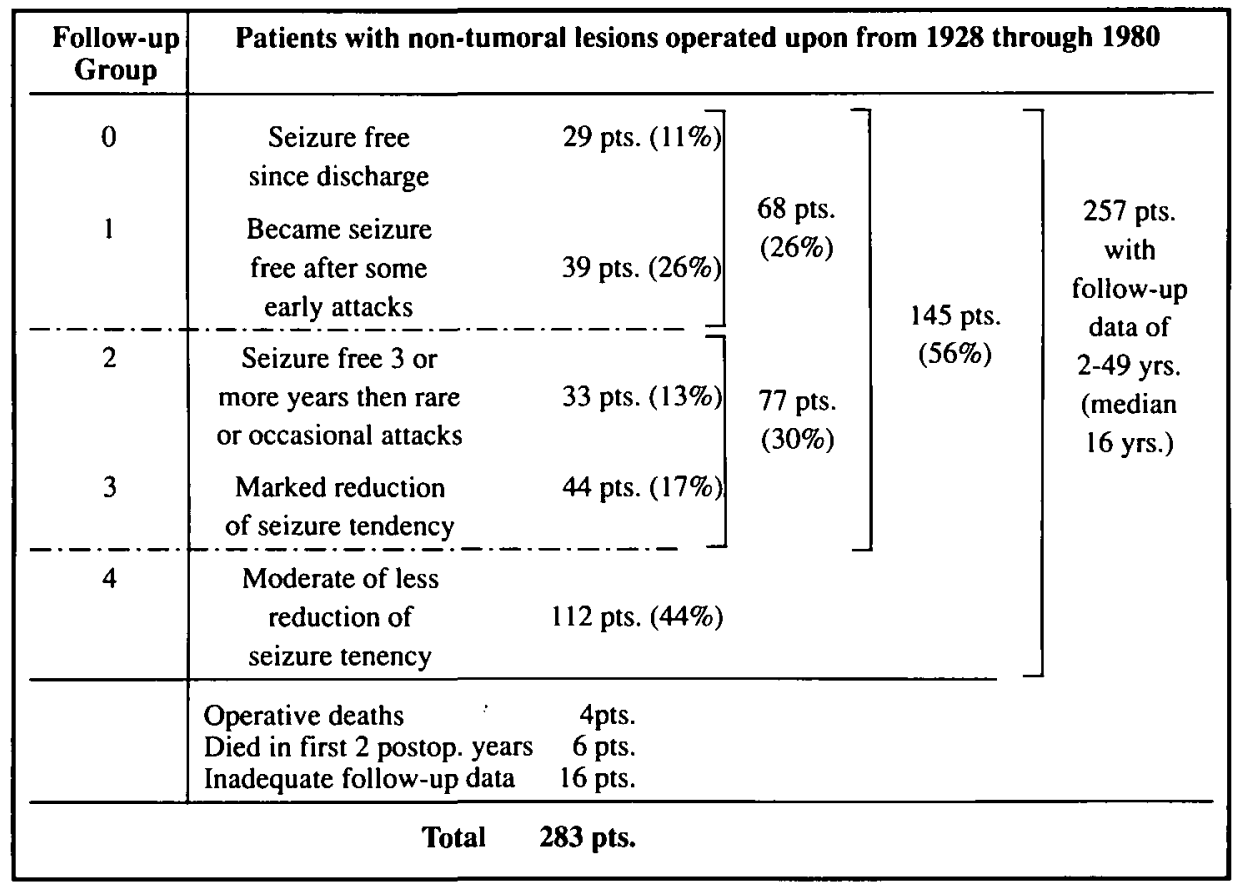

Figure 4 - Frontal Lobe Epilepsy - Results of Cortical Excision 
1 are combined as the seizure free group. Follow-up groups 2 and 3 represent those with a marked reduction of seizure tendency. There are 59 patients in the frontal lobectomy groups with $19(32 \%)$ in the seizure free category. Twenty-two $(47 \%)$ of the 47 patients who had anterior frontal excisions were seizure free. Twelve (35\%) of the 34 patients with smaller convexity excisions were seizure free, as were $9(18 \%)$ of the 50 patients who

\section{Extent of Cortical Resection}

$\begin{array}{lrl}\text { Anterior third of half of frontal lobe } & 18 \text { pts. } & (45 \%) \\ \text { Frontal lobectomy } & 9 \text { pts. } & (22.5 \%) \\ \text { Parasaggital frontal region } & 8 \text { pts. } & (20 \%) \\ \text { Convexity of frontal lobe } & 5 \text { pts. } & (12.5 \%)\end{array}$

Figure 5 - Types of cortical resections carried out in 40 seizure free frontal lobe epilepsy patients (from Rasmussen, reference 22).

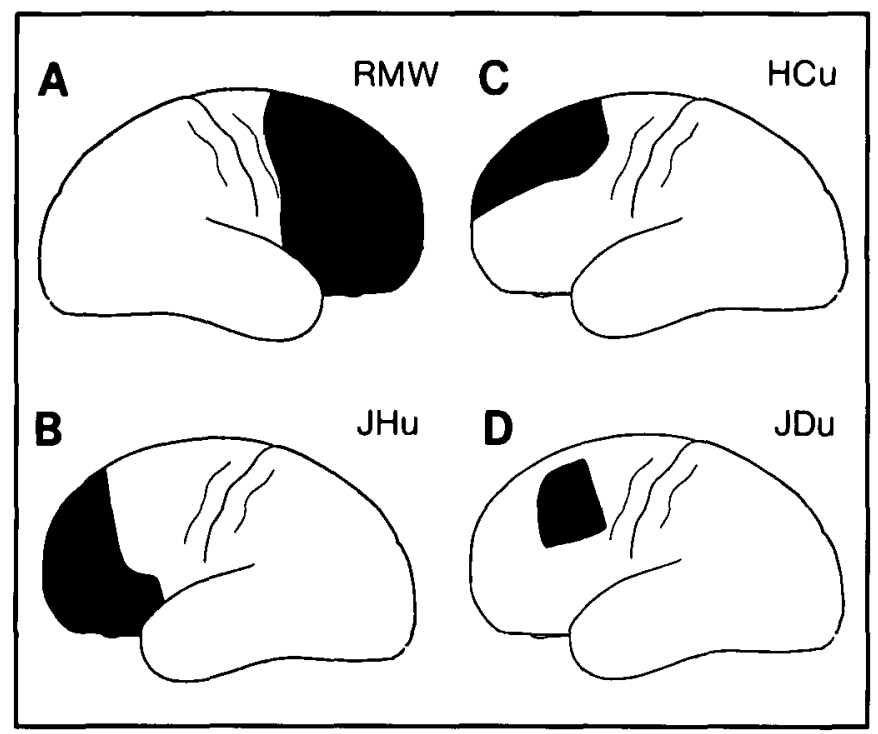

Figure 6-Diagrams of typical frontal lobe cortical excisions made from operative photographs and drawings (from Rasmussen, reference 25)

\begin{tabular}{|lcccc|}
\hline & $\begin{array}{c}\text { FU Groups } \\
\mathbf{0}+\mathbf{1} \\
\text { (seizure } \\
\text { free) }\end{array}$ & $\begin{array}{c}\text { FU Groups } \\
\mathbf{2 + 3} \\
\text { (marked } \\
\text { reduction) }\end{array}$ & $\begin{array}{c}\text { FU Group } \\
\mathbf{4} \\
\text { (moderate } \\
\text { to no } \\
\text { reduction) }\end{array}$ & $\begin{array}{c}\text { Number of } \\
\text { Patients }\end{array}$ \\
\hline Frontal Lobectomy & $19(32 \%)$ & $19(32 \%)$ & $21(36 \%)$ & $59(100 \%)$ \\
Anterior Frontal & $22(47 \%)$ & $13(27 \%)$ & $12(25 \%)$ & $47(100 \%)$ \\
Convexity & $12(35 \%)$ & $7(21 \%)$ & $15(44 \%)$ & $34(100 \%)$ \\
Parasaggital & $9(18 \%)$ & $18(36 \%)$ & $23(46 \%)$ & $50(100 \%)$ \\
Frontal Plus & $6(10 \%)$ & $15(24 \%)$ & $42(66 \%)$ & $63(100 \%)$ \\
\hline Total pts. & $\mathbf{6 8}(\mathbf{2 6 \% )}$ & $\mathbf{7 2 ( 2 9 \% )}$ & $\mathbf{1 1 3 ( 4 5 \% )}$ & $\mathbf{2 5 3 ( 1 0 0 \% )}$ \\
\hline
\end{tabular}

Figure 7 - Frontal Lobe Epilepsy - Results of Various Types of Cortical Excisions. 253 patients with non-tumoral epileptogenic lesions operated upon 1928 through 1980 (from Rasmussen, reference 27. The numbers vary slightly from those in Figure 4 since this analysis was carried out 2 years prior to that in Figure 4) had parasaggital excisions. Only $6(10 \%)$ of the 63 patients in the "frontal plus" group were seizure free.

Follow-up examinations and periodic reports from patients, relatives and physicians over the years have permitted repeated reviews of the files of most of these patients. The accumulated data are not complete and await detailed analysis, but in many instances the clinical and/or the EEG evidence strongly suggests that in those patients who have not had a complete or nearly complete reduction of the seizure tendency the persisting seizure tendency has continued to involve the operated hemisphere. In some patients the proximity of the sensorimotor or speech cortex has played a major role in failure to achieve as good a reduction of the seizure tendency as was hoped for. In other patients it is difficult to evaluate the relative importance of an inadequate determination of the total extent of the potentially epileptogenic cortex, the second order localization, as opposed to an imperfect estimate of the extent of the cortical excision required, the third order localization of the patients' epileptogenic mechanisms.

The increasing use of chronic monitoring to document the patients' seizure patterns plus the progressive sophistication of EEG technology should result in steady improvement in effectiveness of surgical efforts to alleviate medically refractory frontal lobe epilepsy.

\section{REFERENCES}

1. Penfield $W$. The radical treatment of traumatic epilepsy and results of radical operation. Can Med Assoc 1930; 23: 189-197.

2. Penfield $W$. The structural basis of traumatic epilepsy and results of radical operation. Brain 1930; 53: 99-119.

3. Penfield W, Gage L. Cerebral localization of epileptic manifestations. Arch Neurol Psychiatry 1933; 30: 1-19.

4. Penfield W, Evans J. The frontal lobe in man: a clinical study of maximum removals. Brain 1935; 58: 115-133.

5. Hebb DO, Penfield W. Human behavior after extensive bilateral removal from the frontal lobes. Arch Neurol Psychiatry 1940; 44: 421-438.

6. Penfield W, Kristiansen K. Epileptic Seizure Patterns. Springfield: Charles C Thomas, 1950.

7. Penfield W, Rasmussen T. Vocalization and arrest of speech. Arch Neurol Psychiatry 1949; 61: 21-27.

8. Penfield W, Rasmussen T. Cerebral Cortex of Man. MacMillan, New York: MacMillan, 1950.

9. Penfield W, Weich $K$. The supplementary motor area of the cerebral cortex. Arch Neurol Psychiatry 1951; 66: 289-317.

10. Penfield W, Jasper HH. Epilepsy and the Functional Anatomy of the Human Brain. Boston: Little, Brown \& Co., 1954.

11. Rasmussen T. Surgical therapy of frontal lobe epilepsy. Epilepsia 1963; 4 : 181-198.

12. Perot $P$, Weir $B$, Rasmussen $T$. Tuberous sclerosis: surgical therapy for seizures. Arch Neurol 1966; 15: 498-506.

13. Rasmussen $T$. The role of surgery in the treatment of focal epilepsy. In: Ojeman RG, ed. Clinical Neurosurgery. Baltimore: Williams and Wilkins, 1969: 288-311.

14. Rasmussen T. The neurosurgical treatment of focal epilepsy. In: Niedermeyer E, ed. Modern Problems Pharmacopsychiatry. Epilepsy, Vol. 4. Basel/New York: Karger, 1970: 306-325.

15. Rasmussen T, Mathieson G, Leblanc F. Surgical therapy of typical and a forme fruste variety of the Sturge-Weber syndrome. Archives Suisses de Neurologie, Neurochirurgie et de Psychiatrie 1972; 111: 393-409.

16. Rasmussen T. Posttraumatic epilepsy. In: Feiring EH, ed. Brock's Injuries of the Brain and Spinal Cord. New York: Springer, 1974: 544-569.

17. Rasmussen T. Seizures with local onset and elementary symptomatology. In: Vinken PJ, Bruyn GW, eds. Handbook of Clinical 
Neurology, Vol. 15. Amsterdam: North Holland Publishing Co., 1974: 74-86.

18. Rasmussen T. Cortical excision for medically refractory focal epilepsy. In: Harris P, Mawdsley C, eds. The Natural History and Management of Epilepsy. Edinburgh: Churchill Livingston, 1974: 227-239.

19. Rasmussen T. Surgical treatment of epilepsy. In: Tower DB, ed. The Nervous System, Vol. 2. The Clinical Neurosciences, New York: Raven Press 1975: 277-286.

20. Rasmussen T. Surgery of frontal lobe epilepsy. In: Purpura DP, Penry JK, Walter RD, eds. Advances in Neurology, Vol. 8. Neurosurgical Management of the Epilepsies. New York: Raven Press 1975: 197-205.

21. Rasmussen T. Surgical aspects. In: Blau ME, Rapin I, Kinsbourne $M$, eds. Topics in Child Neurology (First International Congress of Child Neurology). Jamaica, New York: Spectrum Publications 1977: 143-157.

22. Rasmussen T, Milner B. The role of early left brain injury in determining lateralization of cerebral speech functions. In: Dimond SJ, Blizard DA, eds. Evolution and Lateralization of the Brain, Annals N.Y. Acad. Sci, Vol. 229, New York: New York Academy of Sciences 1977: 355-369.
23. Rasmussen T. Cortical resection for medically refractory focal epilepsy: results, lessons and questions. In: Rasmussen T, Marino R, eds. Functional Neurosurgery. New York: Raven Press 1979: 253-269.

24. Rasmussen T. Characteristics of a pure culture of frontal lobe epilepsy. Epilepsia 1983; 24: 482-493.

25. Rasmussen T. Commentary: extratemporal cortical excisions and hemispherectomy. In: Engel J, ed. Surgical Treatment of the Epilepsies. New York: Raven Press 1987: 417-424

26. Rasmussen T, Penfield W. Movement of the head and eyes from stimulation of the human frontal cortex. In: The Frontal Lobes, A.R.N.M.D. Vol 27. Baltimore: Williams \& Wilkins, 1947: 346361.

27. Rasmussen T. The MNI frontal lobe surgical seizure series. I: 1929 1980. In: Chauvel P, Delgado-Escueta AV, Bancand J, Halgren E, eds. Frontal Lobe Seizures and Epilepsies. New York: Raven Press (in press).

28. Rasmussen T. How large are frontal lobe epileptogenic zones? I: Surgical aspects. In: Chauvel P, Delgado-Escueta AV, Bancand J, Halgren E, eds. Frontal Lobe Seizures and Epilepsies. New York: Raven Press (in press). 